\title{
Is language impairment more common than executive dysfunction in amyotrophic lateral sclerosis?
}

\author{
Lorna J Taylor, ${ }^{1,2}$ Richard G Brown, ${ }^{1,2}$ Stella Tsermentseli, ${ }^{1,3}$ Ammar Al-Chalabi, ${ }^{2,4}$ \\ Christopher E Shaw, ${ }^{2,4}$ Catherine M Ellis, ${ }^{5}$ P Nigel Leigh, ${ }^{6}$ Laura H Goldstein ${ }^{1,2}$
}

\begin{abstract}
- Additional data are published online only. To view these files please visit the journal online (http://dx.doi. org/10.1136/jnnp-2012303526).

${ }^{1}$ King's College London, Institute of Psychiatry, Department of Psychology, London, UK

${ }^{2}$ King's College London, KHP Centre for Neurodegeneration Research, Institute of Psychiatry, London, UK ${ }^{3}$ Department of Psychology and Counselling, School of Health and Social Care, University of Greenwich, London, UK ${ }^{4}$ King's College London, Institute of Psychiatry, Department of Clinical Neuroscience, London, UK ${ }^{5}$ King's MND Care and Research Centre, King's College Hospital NHS Foundation Trust, London, UK ${ }^{6}$ Trafford Centre for Biomedica Research, Brighton and Sussex Medical School, University of Sussex, Falmer, UK
\end{abstract}

\section{Correspondence to} Professor Laura H Goldstein, Department of Psychology, Institute of Psychiatry, King's College London, PO 77, De Crespigny Park, London SE5 8AF, UK; laura.goldstein@kcl.ac.uk

Received 21 June 2012 Accepted 3 September 2012 Published Online First 2 October 2012

\section{SLinked}

- http://dx.doi.org/10.1136/ jnnp-2012-303851

To cite: Taylor $L$, Brown RG, Tsermentseli S, et al. J Neurol Neurosurg Psychiatry 2013:84: 494-498.

\begin{abstract}
Background Systematic explorations of language abilities in patients with amyotrophic lateral sclerosis (ALS) are lacking in the context of wider cognitive change.
\end{abstract}

Methodology Neuropsychological assessment data were obtained from 51 patients with ALS and 35 healthy controls matched for age, gender and IQ. Composite scores were derived for the domains of language and executive functioning. Domain impairment was defined as a composite score $\leq 5$ th centile relative to the control mean. Cognitive impairment was also classified using recently published consensus criteria. Results The patients with ALS were impaired on language and executive composite scores. Language domain impairment was found in $43 \%$ of patients with ALS, and executive domain impairment in $31 \%$. Standardised language and executive composite scores correlated in the ALS group $(r=0.68, p<0.001)$. Multiple regression analyses indicated that scores on the executive composite accounted for $44 \%$ of the variance in language composite scores.

Conclusions Language impairments are at least as prevalent as executive dysfunction in ALS. While the two domains are strongly associated, executive dysfunction does not fully account for the profile of language impairments observed, further highlighting the heterogeneity of cognitive impairment in non-demented patients with ALS.

\section{INTRODUCTION}

The clinical presentation of amyotrophic lateral sclerosis (ALS) extends beyond the motor system, and studies have reported cognitive impairments of varying severity. While a minority of patients meet criteria for the frontotemporal dementia (FTD) and/or primary progressive aphasia (PPA) subtypes of frontotemporal lobar degeneration (FTLD), ${ }_{1}^{1}$ approximately one-third show cognitive impairments of a milder nature. ${ }^{2-4}$ Executive dysfunction, a negative prognostic indicator of survival in ALS, ${ }^{5}$ has been the most commonly investigated cognitive domain in non-demented patients with ALS. Studies have often reported impairments in fluency, set-shifting, attention and inhibition. ${ }^{6-8}$ Recent consensus criteria have defined cognitive impairment in ALS on the basis of impaired scores $(\leq 5$ th centile of age- and education-matched controls) on at least two measures of executive functioning. ${ }^{3}$ Cognitive impairments in ALS are not, however, restricted to the executive domain. ${ }^{4}$ A number of studies have reported impairments on tasks of language function including confrontation naming, single word and syntactic comprehension, and verb processing. ${ }^{4} 910$ It remains unclear whether this reflects an aphasia-like impairment ${ }^{11}$ or is secondary to executive dysfunction. ${ }^{12}$ There have been comparatively few investigations of language in non-demented patients with ALS and consequently the nature and frequency of language impairment occurrence has not been fully characterised. This study aimed to investigate the prevalence and profile of language change in ALS and the relationship with executive function.

\section{MATERIALS AND METHODS \\ Participants}

Patients were recruited primarily from The King's Motor Neurone Disease (MND) Care and Research Centre, London, UK. Additional patients were recruited from MND Care and Research Centres in Cambridge, Newcastle and Manchester, UK. Healthy control participants were recruited through a volunteer database (http://mindsearch.iop.kcl.ac.uk/). Ethics approval was obtained from King's College Hospital Research Ethics Committee (07/H0808/156). Appropriate institutional approvals were obtained to permit recruitment from additional centres. Informed written consent was obtained from all participants, consistent with the Declaration of Helsinki.

Exclusion criteria for all participants were not having English as a first language, cerebrovascular disease, hypertension, diabetes, history of head injury and current prescription of psychoactive medication. We included right-handed patients, aged $<75$ years with evidence of probable or definite ALS. ${ }^{13}$ Additional patient exclusion criteria included respiratory weakness (forced vital capacity $<70 \%$ ), familial history of ALS, and a clinical diagnosis of comorbid dementia. Where recruited patients showed clinically evident behavioural and/ or cognitive impairment at the initial interview, FTLD diagnostic criteria ${ }^{14}$ were applied to identify patients with FTD, PPA (either progressive nonfluent aphasia or semantic dementia). Patients meeting criteria were excluded.

Domains of language and executive functioning were assessed (table 1) as part of a broader battery of tests including memory function (see online supplementary material). Current and premorbid IQ were assessed using the Wechsler Abbreviated Scale 
Table 1 Test indices included in cognitive composites

\begin{tabular}{|c|c|}
\hline Domain & Measures contributing to the composite \\
\hline Executive functioning & 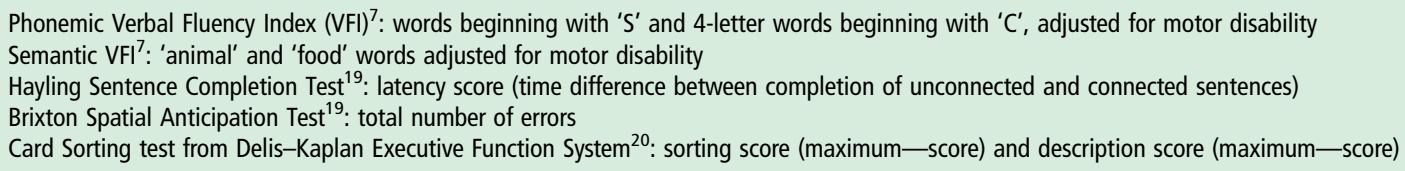 \\
\hline Language & $\begin{array}{l}\text { Cookie Theft Picture (from the Boston Diagnostic Aphasia Examination) }{ }^{21}: \text { Complexity Index } \\
\text { British Picture Vocabulary Scale- } I^{22}: \text { total number of errors } \\
\text { Test of Reception of Grammar-second edition- } 2^{23}: \text { number of blocks failed } \\
\text { Boston Naming Test }{ }^{24}: \text { total number of errors } \\
\text { Category Specific Names Test }{ }^{25}: \text { total number of errors } \\
\text { Oral and Written Naming of Nouns and Verbs }{ }^{26}: \text { total number of errors } \\
\text { Judgement of Synonyms Subtest from Psycholinguistic Assessment of Language Processing in Aphasia }{ }^{27}: \text { total number of errors } \\
\text { Graded Difficulty Spelling Test }{ }^{28}: \text { total number of errors } \\
\text { Pyramids and Palm Trees }{ }^{29}: \text { total number of errors } \\
\text { Kissing and Dancing Test }{ }^{30}: \text { total number of errors } \\
\text { Spot the Word Test }{ }^{31}: \text { total number of errors }^{\text {total }}\end{array}$ \\
\hline
\end{tabular}

of Intelligence ${ }^{15}$ and Wechsler Test of Adult Reading (WTAR), ${ }^{16}$ respectively. The Hospital Anxiety and Depression Scale $(\mathrm{HADS})^{17}$ measured symptoms of anxiety and depression. In the patients, functional abilities were assessed using the Amyotrophic Sclerosis Functional Rating Scale Revised (ALSFRS-R). ${ }^{18}$ Patients were assessed in their homes, and control participants at the Institute of Psychiatry. Assessments were conducted over two sessions, approximately 1 week apart. A fixed test order was used to attempt to control for effects of fatigue and between-test interference across the two groups.

To avoid undertaking multiple between-group comparisons using individual tests, composite scores were calculated from selected indices for the language and executive domains (table 1). As successful completion of the assessment measures may depend on a combination of executive and linguistic abilities (eg, Hayling Sentence Completion Test, phonemic and semantic verbal fluency), total domain score-individual test score correlations were examined to determine the allocation of measures to cognitive composite scores. Standardised $\mathrm{z}$ scores for each test were calculated by subtracting the mean score of controls from the participant's score and then dividing the difference by the control group SD. All measures were adjusted such that a negative score indicated worse performance. $\mathrm{z}$ Scores in each domain were summed, and divided by the number of tests completed to give the composite score. Impairment was defined as a composite score $\leq 5$ th centile $(\sim 1.64$ SDs) below the control mean. Raw scores were used for secondary analyses to consider the variation in impairment across individual measures. As an alternative indicator of overall impairment, the consensus criteria requiring impairment on two or more tests of executive function were also applied, and extended to include classification of language impairment following impairment evident on two or more tests of language functioning.

\section{Statistical analysis}

The data were analysed using SPSS V.17. Demographic, clinical and cognitive characteristics were reported as percentages for categorical data and means for continuous variables. Between-group comparisons were undertaken using $\mathrm{t}$ tests or multivariate analysis of covariance. Categorical data were analysed using $\chi^{2}$ tests. Pearson's correlations and multiple regression analyses were used to examine the relationships between executive and language composite scores. Any outliers were identified and transformed to reduce their impact on the analysis. All tests were two-tailed, and statistical significance was set at $\mathrm{p}<0.05$.

\section{RESULTS}

\section{Demographic and clinical characteristics}

Fifty-one patients with ALS and 35 healthy controls participated in the study. There were no significant differences between the groups with respect to gender distribution, age and years of education, current IQ or estimated premorbid IQ (table 2). Six patients with ALS were unable to complete the WTAR because of dysarthria, so premorbid IQ estimates were obtained for 45 patients with ALS. Patients had higher mean anxiety, depression and total HADS scores than controls, and the HADS total score was used as a covariate for subsequent between-group analyses. However, although participants' HADS scores were not

Table 2 Demographic characteristics of participants

\begin{tabular}{|c|c|c|c|c|}
\hline & \multicolumn{2}{|l|}{ Mean (SD) } & \multirow[b]{2}{*}{$t(d f) / \chi^{2}(d f)$} & \multirow[b]{2}{*}{ p Value } \\
\hline & ALS $(n=51)$ & Controls $(n=35)$ & & \\
\hline Age (years) & $59.84(9.06)$ & $59.31(7.57)$ & $0.28(84)$ & 0.777 \\
\hline Education (years) & $13.75(3.25)$ & $14.71(2.85)$ & $-1.42(86)$ & 0.157 \\
\hline HADS Depression & $4.63(3.36)$ & $1.80(2.05)$ & $4.50(84.39)$ & $<0.001$ \\
\hline HADS Anxiety & $3.67(2.63)$ & $1.69(1.51)$ & $4.02(84)$ & $<0.001$ \\
\hline HADS Total & $8.33(5.80)$ & $3.49(3.30)$ & $4.46(84)$ & $<0.001$ \\
\hline Current IQ (WASI) & $119.21(10.58)$ & $121.06(5.46)$ & $-0.97(62.29)$ & 0.334 \\
\hline Premorbid IQ (WTAR) & $110.33(6.49)$ & $111.22(4.08)$ & $-1.94(75.03)$ & 0.266 \\
\hline Gender, N (\%) male & $29(57 \%)$ & $20(57 \%)$ & $0.00(1)$ & 0.979 \\
\hline
\end{tabular}


modified to account for physical impairment, ${ }^{32}$ the scores did not indicate clinically relevant levels of depression or anxiety.

The mean ALSFRS-R score at the time of neuropsychological assessment was 33.15 (SD 9.07). Seventy-five per cent of patients had limb-onset and 25\% bulbar-onset disease, consistent with typical phenotype frequencies. The average time since onset of symptoms was less than 3 years, with an average delay from symptom onset to diagnosis of $\sim 12$ months. Seventy per cent of patients with ALS were taking riluzole at the time of assessment.

\section{Profile and prevalence of executive and language impairment}

Multivariate analysis of covariance, with HADS total score entered as a covariate, revealed that the patient mean scores were significantly lower overall (more impaired) $(F(4,79)=4.47$, $\mathrm{p}<0.05)$. Univariate contrasts revealed that the mean patient scores were significantly lower for the executive domain $(-0.42$, $\mathrm{SD}=0.70$ compared with $0.0, \mathrm{SD}=0.37$ for controls; $F(1,83)$ $=5.67, \mathrm{p}=0.02$, partial $\mathrm{eta}^{2}=0.064$ ) and for the language domain $(-0.98, \mathrm{SD}=1.31$ compared with $0.0, \mathrm{SD}=0.53$ for controls; $F(1,83)=8.42, \mathrm{p}=0.005$, partial eta $\left.{ }^{2}=0.092\right)$.

Based on the $5 \%$ cut-off derived from the control sample, $31 \%$ of the patients with ALS were classified as impaired on the executive composite and $43 \%$ on the language composite. Of the 23 patients with impairment on the language composite, 10 $(43.5 \%)$ had executive composite scores within the normal range. Pearson's $\chi^{2}$ analysis revealed no relationship between bulbar-onset disease and impairment in the language $\left(\chi^{2}(1)\right.$ $=0.81, \mathrm{p}=0.37)$ and executive domains $\left(\chi^{2}(1)=0.003, \mathrm{p}=0.96\right)$.

Analysis of variance revealed no significant differences in age $\left(F(3,51)=0.462, \mathrm{p}=0.710\right.$, partial eta $\left.{ }^{2}=0.029\right)$, HADS scores $\left(F(3,51)=1.223, \mathrm{p}=0.312\right.$, partial eta $\left.{ }^{2}=0.072\right)$, years of education $\left(F(3,51)=5.67, \mathrm{p}=0.080\right.$, partial eta $\left.{ }^{2}=0.082\right)$, months since onset of illness $(F(3,51)=0.930, \mathrm{p}=0.434$, partial eta $\left.^{2}=0.056\right)$ and ALSFRS-R scores $(F(3,51)=1.70, \mathrm{p}=0.180$, partial eta ${ }^{2}=0.098$ ) between the different cognitive profile groups (no impairment on executive or language composites, impairments on executive and language composites, impairments on the executive composite only, impairment on the language composite only).

Applying the consensus criteria ${ }^{3}$ of impairment on two or more tests of executive function, $13(25 \%)$ patients were classified as cognitively impaired. Applying and extending the criteria to the language tests, 20 (39\%) were impaired. Of these, seven (35\%) performed within the normal range on all measures of executive functioning. Table 3 shows the raw scores on individual language and executive function tests and the proportion of patients meeting criteria for impairment (NB higher scores indicating worse performance). Executive function tests were differentially sensitive to impairment $(6-26.5 \%$ of the patients were found to be impaired on individual measures) as were the language tests, with impairment noted in 4-49\%, depending on the measure.

\section{Relationship between executive and language domains within the ALS group}

To normalise the distributions and equalise variance for within-group analysis, composite scores were restandardised for the ALS participants using the group means and SDs. Pearson's correlations examining associations between composite scores in the ALS group revealed a relationship between the severity of impairment measured by the executive and language $(r=0.68$, $\mathrm{p}<0.001)$ composites. A linear regression analysis indicated that the executive composite scores accounted for $44 \%$ of the variance in the language composite scores (standardised $\beta=0.63$, $95 \%$ CI 0.46 to $0.96, \mathrm{t}(51)=5.80, \mathrm{p}<0.001)$.

\section{DISCUSSION}

The findings of this study are broadly consistent with the extensive literature indicating executive dysfunction in approximately

Table 3 Measures of executive and language functioning: raw scores, SDs and number (and percentage) of patients with ALS impaired on each test

\begin{tabular}{|c|c|c|c|c|c|}
\hline \multirow[b]{2}{*}{ Test } & \multicolumn{2}{|c|}{ Raw score mean $(S D)^{*}$} & \multirow[b]{2}{*}{$F(\mathrm{df})$} & \multirow[b]{2}{*}{$\mathrm{p}$ Value } & \multirow{2}{*}{$\begin{array}{l}\text { No of ALS patients } \\
\text { impaired }(\%)\end{array}$} \\
\hline & ALS & Controls & & & \\
\hline D-KEFS (sorting score) & $1.39(1.46)$ & $0.60(1.03)$ & $5.47(1,82)$ & 0.028 & $13 / 49(26.5)$ \\
\hline D-KEFS (description score) & $39.91(22.25)$ & $36.03(15.05)$ & $0.60(1,82)$ & 0.441 & $3 / 49(6)$ \\
\hline Hayling (latency) & $24.98(22.43)$ & $22.97(21.15)$ & $0.24(1,84)$ & 0.878 & $6(11.5)$ \\
\hline Brixton (errors) & $15.67(4.67)$ & $13.89(3.36)$ & $3.74(1,84)$ & 0.042 & $13(25)$ \\
\hline Phonemic VFI & $8.99(3.92)$ & $7.24(3.03)$ & $3.54(1,84)$ & 0.043 & $13(25)$ \\
\hline Semantic VFI & $2.11(0.99)$ & $1.71(0.95)$ & $1.47(1,84)$ & 0.228 & $5(9.5)$ \\
\hline BNT (errors) & $4.05(4.01)$ & $2.37(1.93)$ & $1.37(1,84)$ & 0.024 & $13(25)$ \\
\hline TROG (blocks failed) & $1.98(1.64)$ & $0.91(0.81)$ & $5.79(1,84)$ & 0.018 & $18(35)$ \\
\hline PPT (errors) & $1.45(1.61)$ & $0.74(1.22)$ & $1.98(1,84)$ & 0.162 & 7 (13.5) \\
\hline KDT (errors) & $2.66(2.27)$ & $0.74(1.38)$ & $13.45(1,84)$ & $<0.001$ & $9(17)$ \\
\hline Synonym Judgement (errors) & $3.78(4.32)$ & $2.00(2.07)$ & $5.53(1,84)$ & 0.021 & $10(19)$ \\
\hline Verb-Noun (errors) & $1.06(1.70)$ & $0.72(1.14)$ & $1.18(1,62)$ & 0.295 & $5 / 44(11.5)$ \\
\hline BPVS (errors) & $11.50(7.24)$ & $5.77(2.79)$ & $12.09(1,84)$ & $<0.001$ & $25(49)$ \\
\hline CSNT (errors) & $21.80(14.73)$ & $16.60(7.10)$ & $1.13(1,84)$ & 0.029 & $12(23)$ \\
\hline Cookie Theft Picture (Complexity Index) & $1.88(0.31)$ & $1.79(0.37)$ & $0.94(1,78)$ & 0.759 & $2 / 46(4)$ \\
\hline STW (errors) & $7.70(4.50)$ & $5.06(3.16)$ & $4.74(1.84)$ & 0.032 & $12(23)$ \\
\hline GDST (errors) & $5.58(3.29)$ & $3.57(2.72)$ & $6.32(1,84)$ & 0.014 & $8(15.5)$ \\
\hline
\end{tabular}

ALS, amyotrophic lateral sclerosis; BNT, Boston Naming Test; BPVS, British Picture Vocabulary Scale; CSNT, Category-Specific Names Test; D-KEFS, Delis-Kaplan Executive Function System; GDST, Graded Difficulty Spelling Test; KDT, Kissing and Dancing Test; PPT, Pyramids and Palm Trees Test; VFI, Verbal Fluency Index; TROG, Test for the Reception of Grammar; STW, Spot the Word Test.

*Higher scores reflect greater impairment. In instances where not all participants were able to complete the measure, the number completing the test is reported. 
one-third of non-demented patients with ALS. ${ }^{2-4}$ Based on a composite measure of executive function, we found 31\% of patients in the present sample to be impaired. This study has also highlighted the occurrence of mild language impairments in non-demented patients with ALS and suggests that such deficits may be at least as common as executive dysfunction, with $43 \%$ of patients showing impairment on the composite measure. A similar pattern was observed when extended consensus criteria were used, with impairment of $25 \%$ and $39 \%$ in executive and language domains, respectively.

The significant association between the severity of language and executive dysfunction and the common co-occurrence of impairment in the two domains is consistent with previous research that has interpreted impaired language abilities in nondemented patients with ALS as being a consequence of executive dysfunction rather than of a primary linguistic disorder. ${ }^{7} 12$ Although the precise mechanism by which executive dysfunction affected language is unclear, aspects of linguistic processing have been related to frontal pathology in ALS. ${ }^{33}$ Conversely, impairments in the executive domain noted may partly reflect language dysfunction and be interpreted as a consequence of impaired linguistic functions. While recognising the co-occurrence and association between impairment in the two cognitive domains, the results of this study suggest that the two deficits are, in part, dissociable. The two domains shared only $44 \%$ of variance in terms of performance, and over $40 \%$ of patients impaired on the language composite performed with the normal range on the executive composite, with a similar figure (35\%) when impairment was based on the consensus criteria.

The present findings suggest that cognitive impairment in ALS is heterogeneous, with some patients showing a predominantly dysexecutive profile, others showing a predominantly linguistic profile, and some a mixed pattern. This is similar to the concept of single-domain and multiple-domain mild cognitive impairment seen in the prodromal stages of Alzheimer's disease and other dementias. ${ }^{34}$ It is also consistent with investigations of FTLD in patients with ALS that have highlighted both overlap and independence of FTD and PPA subtypes. ${ }^{35}$

The increasing awareness of mild cognitive change in nondemented patients with ALS has led to the development of brief screening tools ${ }^{36-38}$ and the publication of consensus criteria for optimal assessment of cognitive change. ${ }^{3}$ These criteria recommend that non-demented patients with ALS be considered as cognitively impaired following performance on standard neuropsychological testing $\leq 5$ th centile, compared with age- and education-matched norms, on two or more distinct cognitive tests sensitive to executive functioning. The significance of language impairment, either combined with executive impairment or in isolation, suggests that published screening tools and consensus criteria for diagnosing mild cognitive impairment may insufficiently address the heterogeneity of cognitive change in non-demented patients with ALS. Failure to include language dysfunction in the definition of cognitive change may therefore lead to underestimations of the prevalence rates of cognitive impairment.

In addition to implications for the formal assessment of cognitive change in ALS clinics, the findings are important when the more general awareness and understanding of cognition in ALS are considered. Increasing awareness of cognitive change is of importance to people with ALS and in the care of individuals with ALS, potentially reducing carer and patient anxiety about the presence of notable cognitive deficits. This study suggests that the observed heterogeneity in cognitive change seen in ALS needs to be further accepted in clinical practice to facilitate a model of effective management of patients with ALS that includes consideration of language impairments as well as executive dysfunction.

The findings of this study may inform future neuroimaging and pathological investigations. The observed heterogeneity of cognitive presentation may reflect variable involvement of frontal and temporal structures subserving executive and language functioning. Furthermore, and in consideration of neuroimaging investigations that have noted white matter tract abnormalities in frontotemporal white matter association tracts, ${ }^{33}$ it is also feasible that the cognitive presentation in ALS may be determined by differential degeneration of white matter. Future research combining in vivo neuropsychological assessment, neuroimaging and postmortem pathological investigation is required to determine the pathological processes and neuroanatomical changes in non-demented patients with ALS with varying cognitive presentations. Previous research has highlighted the role of genetic factors in determining the cognitive profile of patients with ALS with mild cognitive change. ${ }^{8}$ Given the present study's identification of differing presentations of cognitive change, future research could investigate the role of genetic risk factors, as has been undertaken in studies of FTLD.

This study has several limitations that may affect the interpretation of the results and generalisations to the wider ALS population, including use of prevalence rather than incidence sampling, and recruiting from specialist centres rather than community settings. This is a limitation of the majority of neuropsychological investigations of ALS, although some population-based studies have also confirmed the heterogeneity of cognitive impairment in ALS. ${ }^{4}$ While our exclusion criteria were important in ensuring that cognitive impairment could not be better explained by comorbid conditions and disease-related factors, the application of such criteria may render the present sample less representative of the ALS population as a whole, but more informative with respect to the effects of ALS on cognition. Owing to the exclusion of participants with a diagnosis of FTLD, the prevalence of language and executive impairment reported in this study relates only to mild impairments in nondemented patients with ALS, and the study is unable to comment on the incidence rates or nature of executive and language impairment of increasing degrees of severity in the wider ALS population.

Interpretations drawn from this study may be limited by the use of cognitive composite scores calculated from several heterogeneous assessment measures. While this avoids type I errors associated with multiple between-group comparisons and allows a focus on overall rates of impairment in executive and language domains, inspection of patient performance on individual measures (presented for the interested reader in table 3) would provide additional insights into the profiles of cognitive impairment seen in non-demented patients with ALS. In addition, the attempt to assess several areas of language functioning means that the language composite score included a greater number of language tests (relative to executive tests included in the executive composite), potentially rendering the study more sensitive to language than executive impairments.

In addition to the language and executive tests, a number of other tests were conducted as part of a comprehensive neuropsychological examination. A comparable memory domain score did not differentiate the ALS and healthy control groups, and the proportion of ALS and healthy control participants who showed impaired performance on the memory composite score did not significantly differ (see online supplementary material). Data were not considered further. The memory composite score 
included indices from a small number of memory assessments (relative to the language and executive composites), and a greater number of memory tests may have increased the study's sensitivity to detect memory impairments.

These limitations notwithstanding, our identification of language impairment that cannot be explained by executive dysfunction in a proportion of patients with ALS raises questions about the future assessment and ascertainment of mild cognitive change in ALS. Our findings also highlight the need to consider revising the current consensus criteria ${ }^{3}$ for the identification of cognitive impairment in ALS.

Acknowledgements The study was supported by the Medical Research Council, the Motor Neurone Disease Association, UK and the Institute of Social Psychiatry. This work was also supported by the European Community's Health Seventh Framework Programme (FP7/2007-2013) (259867 to AA-C and CES). We would also like to acknowledge Dr J Ealing, Dr C Allen and Dr T Williams and the Dementias \& Neurodegenerative Diseases Research Network (DeNDRoN) for facilitating recruitment at each centre. RGB, AA-C, CES and LHG receive salary support from the National Institute for Health Research (NIHR) Dementia Biomedical Research Unit at South London and Maudsley NHS Foundation Trust and King's College London. The views expressed are those of the authors and not necessarily those of the NHS, the NIHR or the Department of Health.

Contributors LJT contributed to the design of the study, the collection of data, analysed the data, and wrote the manuscript. RGB supervised the study and revised the manuscript. ST contributed to the process of recruitment and data collection. AA-C, CES, PNL and CME revised the manuscript from a clinical perspective. LHG supervised the study, was responsible for the conception of the project, and revised the manuscript.

Funding The study was supported by the Medical Research Council, the Motor Neurone Disease Association, UK and the Institute of Social Psychiatry. This work was also supported by the European Community's Health Seventh Framework Programme (FP7/2007-2013) (259867 to AA-C and CES). RGB receives research support from Parkinsons UK, the Department of Health via the National Institute for Health Research (UK), Medical Research Council (UK), Alzheimer Society (UK), Alzheimer Research Trust (UK), and the Edmund I Safra Foundation (Switzerland). AA-C receives research support from the Motor Neurone Disease Association UK, the ALS Association and the ALS Therapy Alliance. CES receives research support from the Motor Neurone Disease Association UK, the Medical Research Council, the Wellcome Trust and Psychiatry Research Trust. PNL has received research support from the Motor Neurone Disease Association and the Wellcome Trust UK. LHG receives research support from Department of Health/National Institute for Health Research (NIHR) UK and the Motor Neurone Disease Association UK. She has also received support from Epilepsy Research UK, the Institute of Social Psychiatry and the Wellcome Trust UK.

Competing interests $\mathrm{AA}-\mathrm{C}$ receives royalties from the publication of The Brain (Oneworld Publications) and The Genetics of Complex Human Diseases (Cold Spring Harbor Laboratory Press for a book). RGB has received travel expenses and honoraria for speaking and educational activities not funded by industry, and for activities funded by the following companies: UCB Pharma, GSK, Abbott Healthcare Products. CES has received travel expenses and honoraria acting as a consultant to GlaxoSmithKline. PNL has received honoraria or consultancy fees and travel expenses from Sanofi Aventis, GlaxoSmithKline (GSK), Acceleron, Cytokinetics, Neuyronova, and is or has been a member of advisory boards for GSK, Acceleron, Cytokinetics, Neuronova. LHG serves on the Scientific Awards Panel for Epilepsy Action (UK), has received travel expenses and honoraria for speaking and educational activities not funded by industry, receives royalties from the publication of Clinical Neuropsychology (Wiley, 2004, 2012) and The Clinical Psychologist's Handbook of Epilepsy (Routledge, 1997).

Ethics approval King' College Hospital Research Ethics Committee.

Provenance and peer review Not commissioned; externally peer reviewed.

\section{REFERENCES}

1 Neary D, Snowden JS, Mann DM, et al. Frontal lobe dementia and motor neurone disease. J Neurol Neurosurg Psychiatry 1990;53:23-32.

2 Ringholz GM, Appel SH, Bradshaw M, et al. Prevalence and patterns of cognitive impairment in sporadic ALS. Neurology 2005;65:586-90.

3 Strong MJ, Grace GM, Freedman M, et al. Consensus criteria for the diagnosis of frontotemporal cognitive and behavioural syndromes in amyotrophic lateral sclerosis. Amyotroph Lateral Scler 2009;10:131-46.

4 Phukan J, Elamin M, Bede P, et al. The syndrome of cognitive impairment in amyotrophic lateral sclerosis: a population-based study. J Neurol Neurosurg Psychiatry 2012;83:102-8.
5 Elamin M, Phukan J, Bede P, et al. Executive dysfunction is a negative prognostic indicator in patients with ALS without dementia. Neurology 2011;76:1263-9.

6 Abrahams S, Goldstein LH, Al Chalabi A, et al. Relation between cognitive dysfunction and pseudobulbar palsy in amyotrophic lateral sclerosis. J Neurol Neurosurg Psychiatry 1997;62:464-72.

7 Abrahams S, Leigh PN, Harvey A, et al. Verbal fluency and executive dysfunction in amyotrophic lateral sclerosis (ALS). Neuropsychologia 2000;38:734-47.

8 Wicks P, Abrahams S, Papps B, et al. SOD1 and cognitive dysfunction in familial amyotrophic lateral sclerosis. J Neurol 2009;256:234-41.

9 Cobble M. Language impairment in motor neurone disease. J Neurol Sci 1998;160 (Suppl 1):S47-52.

10 Grossman M, Anderson C, Khan A, et al. Impaired action knowledge in amyotrophic lateral sclerosis. J Neurol 2008;7:1396-401.

11 Bak TH, Hodges JR. The effects of motor neurone disease on language: further evidence. Brain Lang 2004;89:354-61.

12 Talbot PR, Goulding PJ, Lloyd JJ, et al. Inter-relation between 'classic' motor neuron disease and frontotemporal dementia: neuropsychological and single photon emission computed tomography study. I Neurol Neurosurg Psychiatry 1995:58:541-7.

13 Brooks B, Miller R, Swash $M$, et al. El Escorial revisited: revised criteria for the diagnosis of amyotrophic lateral sclerosis. Amyotroph Lateral Scler 2000;1:293-9.

14 Neary D, Snowden JS, Gustafson L, et al. Frontotemporal lobar degeneration: a consensus on clinical diagnostic criteria. Neurol 1998;51:1546-54.

15 Wechsler D. Wechsler abbreviated scale of intelligence. San Antonio, Texas: Psychological Corporation, 1999.

16 Holdnack J. Wechsler test of adult reading. San Antonio, Texas: Psychological Corporation, 2001

17 Zigmond AS, Snaith RP. The hospital anxiety and depression scale. Acta Psychiatr Scand 1983;67:361-70.

18 Cedarbaum JM, Stambler N, Malta E, et al. The ALSFRS-R: a revised ALS functional rating scale that incorporates assessments of respiratory function. BDNF ALS Study Group (Phase III). J Neurol Sci 1999:169:13-21.

19 Burgess P, Shallice T. The hayling and brixton tests. Bury St Edmonds, UK: Thames Valley Company, 1997.

20 Delis D, Kaplan E, Krammer J. Delis-Kaplan executive function system. San Antonio: Psychological Corporation, 2001.

21 Goodglass H, Kaplan E. The Boston diagnostic aphasia examination. Philadelphia: Lea \& Febiger, 1972

22 Dunn L, Dunn L, Whetton C. The British picture vocabulary scale. 2nd edn. Windsor, UK: NFER-Nelson, 1997.

23 Bishop DV. Test for the reception of grammar (TROG-2). London, UK: Harcourt Assessment, 2003

24 Kaplan E, Goodglass H, Weintrab S. Boston naming test. Philadelphia: Lee \& Febiger, 1983

25 McKenna P. Category specific names test. Hove, UK: Psychology Press, 1997.

26 Hillis $A E$, Oh $S$, Ken L. Deterioration of naming nouns versus verbs in primary progressive aphasia. Ann Neurol 2004:55:268-75.

27 Kay J, Lesser R, Coltheart M. Psycholinguistic assessments of language processing in Aphasia (PALPA). East Sussex, England: Lawrence Erlbaum Associates, 1992.

28 Baxter D. Measuring Dysgraphia: a graded-difficulty spelling test. Behav Neurol 1994;7:101-7.

29 Howard D, Patterson K. Pyramids and palm trees. Bury St Edmonds, UK: Thames Valley Test Company, 1992

30 Bak T, Hodges J. "Kissing and Dancing"-a test to distinguish the lexical and conceptual contributions to noun/verb and action/object dissociation. Preliminary results in patients with frontotemporal dementia. J Neurolinguistics 2003;16:169-81.

31 Baddeley A, Emslie H, Nimmo-Smith I. The Spot-the-Word test: a robust estimate of verbal intelligence based on lexical decision. Br J Clin Psychol 1993;32:55-65.

32 Gibbons CJ, Mills RJ, Thornton EW, et al. Rasch analysis of the Hospital Anxiety and Depression Scale (HADS) for use in motor neurone disease. Health Qual Life Outcomes 2011;9:82-90

33 Tsermentseli S, Leigh PN, Goldstein LH. The anatomy of cognitive impairment in amyotrophic lateral sclerosis: more than frontal lobe dysfunction. Cortex 2012;48:166-82.

34 Peterson RC, Doody R, Kurz A, et al. Current concepts in mild cognitive impairment. Arch Neurol 2001;58:1985-92.

35 Rakowicz WP, Hodges JR. Dementia and aphasia in motor neuron disease: an underrecognised association? J Neurol Neurosurg Psychiatry 1998;65:881-9.

36 Gordon PH, Wang Y, Doorish C, et al. A screening assessment of cognitive impairment in patients with ALS. Amyotroph Lateral Scler 2007;8:362-5.

37 Flaherty-Craig C, Brothers A, Dearman B, et al. Penn State screen exam for the detection of frontal and temporal dysfunction syndromes: application to ALS. Amyotroph Lateral Scler 2009;10:107-12.

38 Woolley SC, York MK, Moore DH, et al. Detecting frontotemporal dysfunction in ALS: utility of the ALS Cognitive Behavioral Screen (ALS-CBS). Amyotroph Lateral Scler 2010;11:303-11. 